Mots. Les langages du politique

Le travail et ses maux

\title{
Romain Colonna, De la minoration à l'émancipation: itinéraires sociolinguistiques
}

Valeria Villa-Perez

\section{OpenEdition}

Journals

Édition électronique

URL : https://journals.openedition.org/mots/28640

DOI : $10.4000 /$ mots. 28640

ISSN : 1960-6001

Éditeur

ENS Éditions

Édition imprimée

Date de publication : 8 juillet 2021

Pagination : 127-130

ISBN : 979-10-362-0478-4

ISSN : 0243-6450

Référence électronique

Valeria Villa-Perez, "Romain Colonna, De la minoration à l'émancipation : itinéraires sociolinguistiques », Mots. Les langages du politique [En ligne], 126 | 2021, mis en ligne le 15 juin 2021, consulté le 17 juin 2021. URL : http://journals.openedition.org/mots/28640 ; DOl : https://doi.org/10.4000/mots.28640

Ce document a été généré automatiquement le 17 juin 2021

(c) ENS Éditions 


\title{
Romain Colonna, De la minoration à l'émancipation : itinéraires sociolinguistiques
}

\author{
Valeria Villa-Perez
}

\section{RÉFÉRENCE}

Romain Colonna, De la minoration à l'émancipation : itinéraires sociolinguistiques, Ajaccio, Albiana, 2020, 208 p.

1 En prenant appui sur le terrain corse, l'ouvrage de Romain Colonna, paru aux éditions Albiana dans la collection "Carta», met la focale sur les différentes facettes de la minoration (socio)linguistique, tour à tour décrites, analysées, déconstruites au fil de dix chapitres. Ces derniers, parus une première fois entre 2012 et 2020, dans des supports éditoriaux divers, ont été efficacement assemblés et précédés par une longue introduction. C'est ici que commence l'itinéraire sociolinguistique de R. Colonna, qui se structure et se développe à partir d'un triptyque conceptuel qui lui est cher: la domination, la minoration et l'émancipation. À ce propos, avant de passer en revue une sélection de cinq chapitres, nous nous arrêterons quelques instants sur un aspect intéressant mis en exergue par le titre de l'ouvrage. En effet, si la notion de minoration a été davantage explorée dans la littérature, la conceptualisation de ses voies de sortie - ici dénommée émancipation - l'a sans doute été un peu moins. De ce fait, il s'agit à notre sens de la structure notionnelle forte de ce livre, d'une part en raison de sa place et de ses perspectives dans la réflexion et le positionnement du sociolinguiste, et d'autre part en raison du fait qu'elle nous semble aller de pair avec une vision engagée de ce champ disciplinaire, ayant pour but de provoquer un "impact » dans et pour le tissu social à travers l'apport de la recherche scientifique.

2 Peut-il en être autrement pour un chercheur en contexte minoritaire ? Le cadre est posé d'emblée par R. Colonna qui avance : « il ne saurait y avoir dans le milieu qui est le 
nôtre une recherche asociale, apolitique, aseptisée, décontextualisée et, inversement, il ne saurait y avoir de visée émancipatrice pour une communauté sociolinguistique, [...], sans que celle-ci ne puisse trouver appui, [...], sur un discours scientifique [...] rigoureux et structuré » (p.12). R. Colonna soumet à la réflexion des points fondamentaux qui constituent depuis toujours des priorités pour la sociolinguistique (comme pour d'autres disciplines), parmi lesquelles l'ancrage social, la subjectivité assumée par le chercheur ou le devoir de restitution de la recherche (p. 11, 15).

3 Venons-en maintenant à la présentation de quelques thématiques de l'ouvrage qui inscrivent le triptyque conceptuel cité plus haut sur plusieurs niveaux, du macro au micro.

4 Notre parcours commencera avec le cinquième chapitre qui se fonde sur l'étude des textes et politiques linguistiques européens afin d'y analyser le traitement, ou le nontraitement, des langues minorées. Les langues "non étatisées» (régionales et/ou minoritaires) ont en effet un statut particulier relevant des politiques des États. Ainsi, comme le souligne R. Colonna, les intentions de l'Union européenne sur la diversité linguistique en restent à un stade déclaratif et ne sont pas accompagnées d'actes concrets en faveur des langues minorées, si on exclut la Charte européenne des langues régionales ou minoritaires, dont la ratification aurait un poids limité pour le corse par exemple. R. Colonna rappelle ensuite que la permanence d'une hiérarchisation entre langues officielles et non officielles engendre l'exclusion de ces dernières « du droit et du champ de la reconnaissance » (p. 107).

5 Le sixième chapitre revient sur la notion de «langue de France » en soulignant le caractère non anodin de toute pratique de dénomination. L'appellation en question instaure une relation de propriété sur les langues régionales et opère une simplification homogénéisante, excluant à la fois les spécificités et l'enracinement territorial de ces langues. Cela conduit R. Colonna à (re)définir cette catégorisation d'une part comme un processus de déterritorialisation et, d'autre part, comme une forme de patrimonialisation et de nationalisation rappelant, selon une logique économique et régulatrice, ce que le chercheur décrit comme une « Opération Publique de Rachat » mise en œuvre par l'État français.

6 Dans le huitième chapitre, l'auteur replace la question du corse dans le domaine éducatif et met en évidence et en cause les effets contradictoires du «libéralisme glottopolitique» (p.147). R. Colonna précise que l'enseignement du corse n'est pas obligatoire ; autrement dit « obligation est faite à l'État d'assurer l'enseignement alors que l'individu est dispensé de suivre obligatoirement ce même enseignement » (p. 141). L'auteur dénonce les effets pervers de la «hiérarchisation discriminante des disciplines» (p.142) et, par conséquent, de l'optionalité du corse dans le système scolaire, laquelle accentue sa minoration puisque, s'agissant d'une langue dont le rayonnement est limité, elle devient de facto moins attrayante pour les élèves.

7 En s'appuyant sur le cadre théorique de l'analyse du discours et de la philosophie politique, le neuvième chapitre, par l'analyse de trois allocutions ou déclarations politiques, celles d'Emmanuel Macron, de Jean-Luc Melenchon et de Michel Barat (un ancien recteur de l'académie de Corse), élucide certains déséquilibres sociaux et linguistiques liés aux langues régionales et au corse en particulier. Ces déséquilibres sont à imputer, d'après R. Colonna, à la « dimension universelle conférée par le pouvoir politique à la culture et langue françaises» (p. 154). Les indices d'une dimension universelle repérés dans les trois discours révèlent la permanence, la récurrence et la 
reproduction d'une idéologie monolingue et universaliste qui repose sur «la tension minoration/majoration» (p. 166).

8 Le dixième chapitre, qui clôt l'ouvrage, approfondit les questionnements autour de la minoration et des fonctionnements diglossiques à l'aune de la notion d'auto-odi (la haine de soi), concept élaboré par le sociolinguiste catalan Rafael Ninyoles qui explique ainsi le remplacement de la langue propre par la langue dominante. R. Colonna s'intéresse au rôle des représentations, idéologies et attitudes dans le long processus, aux niveaux individuel et collectif, de substitution linguistique et d'interruption de la transmission intergénérationnelle, ainsi qu'aux problématiques identitaires qui s'ensuivent. Il conceptualise cette relation du locuteur à la langue minorée en l'expliquant aussi en termes d'« ambivalence solidaire» (p. 171); autrement dit, les rapports ambigus qu'un locuteur entretient avec une langue minorée suivraient un double mouvement allant de l'idéalisation de la langue à l'(auto)dénigrement. R. Colonna précise enfin que les fonctionnements diglossiques évoluent vers une nouvelle forme de culpabilité du locuteur: si, par le passé, il se sentait coupable de parler une langue stigmatisée, il déplore aujourd'hui de ne plus parler sa langue de cœur.

9 Le cadre décrit et analysé à partir de l'exemple insulaire pousse R. Colonna à plaider pour un rééquilibrage social et linguistique des situations de minoration et à assumer pleinement une "forme d'application-implication du sociolinguiste, au risque de réduire la frontière qui sépare le scientifique du politique» (p. 23), cadre dans lequel « la politique doit être envisagée par rapport à son sens large, à savoir ce qui a trait à l'organisation de la société, donc à l'organisation des langues » (loc. cit.).

10 Au-delà des nombreux éclairages fournis pour le cas corse, la lecture de ce livre est d'un grand intérêt pour l'étude d'autres contextes et langues minorées. L'apport conceptuel stimulant de la «sociolinguistique programmatique» (p.12) de R. Colonna, en plaçant la dimension politique au centre de la réflexion, permet au lecteur de comprendre des questions et des enjeux complexes relatifs à la minoration, à la fois en tant que mécanisme et processus, et d'explorer des voies d'issue possibles, par exemple des actions réellement poussées visant "l'autonomie glottopolitique et langagière » (p. 60-63) de la langue minorée.

11 En insistant sur le double ancrage du fait langagier comme fait social et, par conséquent, comme fait politique, ce livre envisage la sociolinguistique comme une science politique. Dès lors, il n'est pas sans intérêt de revenir sur le versant étymologique du terme convoqué : en grec, l'adjectif politique ( $\pi 0 \lambda \imath \tau \imath \kappa o ́ s)$ et le mot cité ( $\pi$ ódı) partagent la même racine ( $\pi \circ \lambda$-) que l'expression qui désigne la «multitude » (oí $\pi \circ \lambda \lambda \circ \imath^{1}$. Transposer la question de la pluralité sous l'angle (socio)linguistique signifie prendre en compte les spécificités de cette multitude de locuteurs en tant qu'entité collective non homogène ; transposer la même question dans la perspective d'un projet de société linguistique et éducatif inclusif impliquerait de veiller à préserver la diversité et la singularité des locuteurs. Il s'agit à notre sens de l'impression globale qui prévaut à la lecture de cet ouvrage percutant. 


\section{NOTES}

1. Voir l'entrée «politica » dans Dizionario di filosofia, Rome, Istituto della enciclopedia italiana, 2009,

https://www.treccani.it/enciclopedia/politica_\%28Dizionario-di-filosofia\%29/

(consulté le 07/04/2021).

\section{AUTEURS}

\section{VALERIA VILLA-PEREZ}

Université Jean Monnet Saint-Étienne, équipe ECCLA 